\title{
Detecting chiral pairing and topological superfluidity using circular dichroism
}

\author{
J. M. Midtgaard, ${ }^{1}$ Zhigang Wu $\odot,{ }^{2,3}$ N. Goldman, ${ }^{4}$ and G. M. Bruun $\odot^{1,2}$ \\ ${ }^{1}$ Department of Physics and Astronomy, Aarhus University, Ny Munkegade, DK-8000 Aarhus C, Denmark \\ ${ }^{2}$ Shenzhen Institute for Quantum Science and Engineering and Department of Physics, \\ Southern University of Science and Technology, Shenzhen 518055, China \\ ${ }^{3}$ Peng Cheng Laboratory, Center for Quantum Computing, Shenzhen 518055, China \\ ${ }^{4}$ Center for Nonlinear Phenomena and Complex Systems, Université Libre de Bruxelles, CP 231, Campus Plaine, B-1050 Brussels, Belgium
}

(Received 25 March 2020; accepted 21 August 2020; published 9 September 2020)

\begin{abstract}
Realizing and probing topological superfluids is a key goal for fundamental science, with exciting technological promises. Here, we show that chiral $p_{x}+i p_{y}$ pairing in a two-dimensional topological superfluid can be detected through circular dichroism, namely, as a difference in the excitation rates induced by a clockwise and counterclockwise circular drive. For weak pairing, this difference is to a very good approximation determined by the Chern number of the superfluid, whereas there is a nontopological contribution scaling as the superfluid gap squared that becomes significant for stronger pairing. This gives rise to a competition between the experimentally driven goal to maximize the critical temperature of the superfluid, and observing a signal given by the underlying topology. Using a combination of strong-coupling Eliashberg and Berezinskii-Kosterlitz-Thouless theory, we analyze this tension for an atomic Bose-Fermi gas, which represents a promising platform for realizing a chiral superfluid. We identify a wide range of system parameters where both the critical temperature is high and the topological contribution to the dichroic signal is dominant.
\end{abstract}

DOI: 10.1103/PhysRevResearch.2.033385

\section{INTRODUCTION}

The realization and manipulation of topological superfluids and superconductors is presently one of the most actively pursued goals in physics. In addition to being interesting from a fundamental science point of view, their Majorana edge modes promise applications for quantum computing [1]. Zeroenergy states at the ends of one-dimensional (1D) nanowires have been observed, consistent with the presence of Majorana modes [2,3]. So far, the observation of topological superfluidity in $2 \mathrm{D}$ is missing. The most promising solid-state candidate for a $2 \mathrm{D}$ topological superconductor is $\mathrm{Sr}_{2} \mathrm{RuO}_{4}$, but the precise symmetry of the order parameter in this crystal remains subject to intense debate [4-6]. It has recently been shown that an atomic 2D Fermi gas immersed in a BEC offers a promising platform for realizing a topological superfluid [7-9]. The fermions form Cooper pairs with chiral symmetry by exchanging sound modes in the BEC, and the system offers sufficient flexibility so that one can tune the superfluid critical temperature to be within experimental reach. Experimentally, such a Bose-Fermi mixture has been realized using ${ }^{173} \mathrm{Yb}-{ }^{7} \mathrm{Li}$ atoms, which constitutes an important step towards an unequivocal realization of a topological $p_{x}+i p_{y}$ superfluid [10].

Published by the American Physical Society under the terms of the Creative Commons Attribution 4.0 International license. Further distribution of this work must maintain attribution to the author(s) and the published article's title, journal citation, and DOI.
A key question concerns the detection of topological superfluidity in atomic gases. Their topological properties are not easily extracted from thermodynamic measurements nor by using common probes such as radio-frequency spectroscopy [11]. Contrary to the chiral edge modes of single-particle band structures, which have been detected in experiments [12], the observation of Majorana states [13,14] is complicated by their small number and their particle-hole nature.

It was recently proposed $[15,16]$ and experimentally demonstrated [17] that the topologically invariant Chern number can be detected in atomic gases through circular dichroism, namely, by analyzing excitation rates upon applying a circular drive. This topological probe was first introduced for noninteracting Chern insulators [15], and later applied to interacting many-body systems [18-20]. Inspired by this approach, we hereby demonstrate that the chirality of the $p_{x}+i p_{y}$ pairing is revealed in the circular dichroism of the superfluid. For weak pairing, the differential excitation rate obtained from opposite drive orientations, integrated over the drive frequency, is shown to be determined by the Chern number of the topological superfluid, in direct analogy with Chern insulators [15]. However, in contrast with the latter case, a nontopological contribution scaling as the superfluid gap squared becomes significant for strong pairing. The resulting competition between maximizing the superfluid critical temperature while detecting a genuine topological signature is analyzed for a concrete atomic Bose-Fermi mixture. Using the strong-coupling Eliasberg equations combined with Berezinskii-Kosterlitz-Thouless (BKT) theory, we identify a wide and accessible parameter regime where the superfluid critical temperature is high and the dichroic signal is 
dominated by the topological Chern number. Our results demonstrate that the dichroic probe offers an experimentally promising pathway to detect topological superfluidity.

\section{TOPOLOGICAL RESPONSES IN SUPERFLUIDS}

We first establish a connection between circular dichroism, the Hall conductivity, and the Chern number of the superfluid. Consider a 2D system of spin-polarized fermions described by the Hamiltonian

$$
\begin{aligned}
H_{0}= & \int d^{2} r \psi^{\dagger}(\mathbf{r})\left(-\frac{\nabla^{2}}{2 m}\right) \psi(\mathbf{r}) \\
& +\frac{1}{2} \iint d^{2} r d^{2} r^{\prime} \psi^{\dagger}(\mathbf{r}) \psi^{\dagger}\left(\mathbf{r}^{\prime}\right) V\left(\mathbf{r}-\mathbf{r}^{\prime}\right) \psi\left(\mathbf{r}^{\prime}\right) \psi(\mathbf{r}),
\end{aligned}
$$

where $\psi(\mathbf{r})$ is the fermion field and $V\left(\mathbf{r}-\mathbf{r}^{\prime}\right)$ is an interaction giving rise to pairing, which may result from a $p$-wave Feshbach resonance or, as we will consider later, from an induced interaction. Within BCS theory, this $p$-wave superfluid can be described by the Hamiltonian

$$
H_{\mathrm{BCS}}=\sum_{\mathbf{k}} \Phi_{\mathbf{k}}^{\dagger} H_{\mathbf{k}} \Phi_{\mathbf{k}}, \quad H_{\mathbf{k}}=\mathbf{h}_{\mathbf{k}} \cdot \boldsymbol{\tau},
$$

where $\Phi_{\mathbf{k}} \equiv\left[a_{\mathbf{k}}, a_{-\mathbf{k}}^{\dagger}\right]^{T}, \quad \mathbf{h}_{\mathbf{k}}=\left(\operatorname{Re} \Delta_{\mathbf{k}},-\operatorname{Im} \Delta_{\mathbf{k}}, \xi_{\mathbf{k}}\right)^{T}$, and where $\boldsymbol{\tau}=\left(\tau_{1}, \tau_{2}, \tau_{3}\right)^{T}$ with $\tau_{i}$ the Pauli matrices. Here, $\xi_{\mathbf{k}}=$ $k^{2} / 2 m-\mu$, where $\mu$ is the chemical potential, and $\Delta_{\mathbf{k}}$ is the gap parameter ( $\hbar=1$ throughout). The latter is taken to have chiral $p$-wave symmetry, i.e., $\Delta_{\mathbf{k}}=\Delta_{k} e^{i \phi}$, where $\phi$ is the polar angle of the momentum $\mathbf{k}$ and $k=|\mathbf{k}|$, since this gives the lowest energy for $p$-wave pairing as it has no nodes [21,22]. Indeed, as $\Delta_{k} \propto k$ for $k \ll k_{F}$ due to Fermi antisymmetry, we get $\Delta_{\mathbf{k}} \propto k_{x}+i k_{y}$. This results in a topological phase characterized by a Chern number $C=-1$ for $\mu>0$ whereas $C=0$ for $\mu<0$ [23]. The Chern number reads

$$
\begin{aligned}
C & =\int \frac{d^{2} k}{4 \pi} \frac{1}{\left|\mathbf{h}_{\mathbf{k}}\right|^{3}} \mathbf{h}_{\mathbf{k}} \cdot \partial_{k_{x}} \mathbf{h} \times \partial_{k_{y}} \mathbf{h} \\
& =\int \frac{d^{2} k}{2 \pi}\left[\frac{v_{x}}{E_{\mathbf{k}}^{3}} \operatorname{Im}\left(\Delta_{\mathbf{k}}^{*} \partial_{k_{y}} \Delta_{\mathbf{k}}\right)+\frac{\xi_{\mathbf{k}}}{2 E_{\mathbf{k}}^{3}} \operatorname{Im}\left(\partial_{k_{x}} \Delta_{\mathbf{k}} \partial_{k_{y}} \Delta_{\mathbf{k}}^{*}\right)\right],
\end{aligned}
$$

where $v_{x}=k_{x} / m$ and $E_{\mathbf{k}}=\sqrt{\xi_{\mathbf{k}}^{2}+\left|\Delta_{\mathbf{k}}\right|^{2}}$ is the BCS quasiparticle energy. The second term in the integrand scales as $\Delta_{\mathbf{k}}^{2} / \mu^{2}$ so that the Chern number can be approximated by the first term in the regime $\Delta_{\mathbf{k}} \ll \mu$.

We now show that the Chern number in Eq. (3) can be extracted from circular dichroism, namely, by monitoring excitation rates upon a circular drive $[15,17]$. We consider a circular drive of the form

$$
V_{ \pm}(\mathbf{r} ; \mathbf{q})=2 \mathcal{E}(x \cos \Omega t \pm y \sin \Omega t) \cos \mathbf{q} \cdot \mathbf{r},
$$

and we will set $\mathbf{q} \rightarrow 0$ at the end of the calculations, corresponding to a uniform circular shaking [17]. This reads

$$
V_{ \pm}(\mathbf{q})=\frac{\mathcal{E}}{i}\left[-\frac{\partial \tilde{n}(\mathbf{q})}{\partial q_{x}} \pm i \frac{\partial \tilde{n}(\mathbf{q})}{\partial q_{y}}\right] e^{-i \Omega t}+\text { H.c. }
$$

in second quantization, where $n(\mathbf{r})=\psi^{\dagger}(\mathbf{r}) \psi(\mathbf{r})$ is the density operator, $n(\mathbf{q})$ is its Fourier transform, and $\tilde{n}(\mathbf{q})=$ $[n(\mathbf{q})-n(-\mathbf{q})] / 2$. Within linear response, the excitation rate out of the ground state of $H_{0}$ induced by $V_{ \pm}(\mathbf{q})$ can be calculated using Fermi's golden rule as

$$
\begin{aligned}
\Gamma_{ \pm}(\mathbf{q}, \Omega)= & 2 \pi \mathcal{E}^{2} \sum_{f} \mid\left\langle f\left|\frac{\partial \tilde{n}(\mathbf{q})}{\partial q_{x}} \pm i \frac{\partial \tilde{n}(\mathbf{q})}{\partial q_{y}}\right| g||^{2}\right. \\
& \times \delta\left(E_{f}-E_{g}-\Omega\right),
\end{aligned}
$$

where $|g\rangle$ and $|f\rangle$ denote the ground and excited states of $H_{0}$ with energy $E_{g}$ and $E_{f}$, respectively.

The observable of interest is provided by the differential integrated rate (DIR), which is defined as [15]

$$
\Delta \Gamma=\lim _{\mathbf{q} \rightarrow 0} \frac{1}{2} \int_{0}^{\infty} d \Omega\left[\Gamma_{+}(\mathbf{q}, \Omega)-\Gamma_{-}(\mathbf{q}, \Omega)\right] .
$$

Substituting Eq. (6) into Eq. (7), we find

$$
\Delta \Gamma=-\pi i \mathcal{E}^{2} \lim _{\mathbf{q} \rightarrow 0}\left\langle g\left|\left[\frac{\partial n(\mathbf{q})}{\partial q_{x}}, \frac{\partial n(-\mathbf{q})}{\partial q_{y}}\right]\right| g\right\rangle,
$$

where we have used momentum conservation to eliminate terms. We now use the continuity equation to write $\Delta \Gamma$ in terms of the density-current correlation function. From $\partial_{t} n(\mathbf{r}, t)+\nabla \cdot \mathbf{j}(\mathbf{r}, t)=0$, we find

$$
\langle g|n(\mathbf{q})| f\rangle=\frac{\langle g|\mathbf{q} \cdot \mathbf{j}(\mathbf{q})| f\rangle}{E_{f}-E_{g}},
$$

where the Fourier transform of the current reads

$$
\mathbf{j}(\mathbf{q})=(1 / 2 m i) \int d^{2} r e^{-i \mathbf{q} \cdot \mathbf{r}}\left[\psi^{\dagger}(\mathbf{r}) \nabla \psi(\mathbf{r})-\text { H.c. }\right] .
$$

Using Eqs. (8) and (9) and noting that

$$
\lim _{q_{y} \rightarrow 0} \lim _{q_{x} \rightarrow 0}\left\langle g\left|\partial_{q_{y}} n(\mathbf{q})\right| f\right\rangle=\lim _{q_{y} \rightarrow 0} \lim _{q_{x} \rightarrow 0}\left\langle g\left|n(\mathbf{q}) / q_{y}\right| f\right\rangle,
$$

we find the relation

$$
\Delta \Gamma / \mathcal{A}=2 \pi \mathcal{E}^{2} \sigma_{x y},
$$

which connects the DIR to the static Hall conductivity

$$
\begin{aligned}
\sigma_{x y} & \equiv \lim _{q_{y} \rightarrow 0} \lim _{q_{x} \rightarrow 0} \lim _{\omega \rightarrow 0} \frac{1}{i \mathcal{A} \omega} \chi_{j_{x}, j_{y}}(\mathbf{q}, \omega) \\
& =\lim _{q_{y} \rightarrow 0} \lim _{q_{x} \rightarrow 0} \lim _{\omega \rightarrow 0} \frac{1}{i \mathcal{A} q_{y}} \chi_{j_{x}, n}(\mathbf{q}, \omega) .
\end{aligned}
$$

Here, $\mathcal{A}$ is the system's area, and $\chi_{A, B}(\mathbf{q}, \omega)$ is the Fourier transform of the retarded correlation function

$$
\chi_{A, B}\left(\mathbf{q}, t-t^{\prime}\right)=-i \theta\left(t-t^{\prime}\right)\left\langle\left[A(\mathbf{q}, t), B\left(-\mathbf{q}, t^{\prime}\right)\right]\right\rangle,
$$

with $\theta(x)$ the Heaviside function. We note that the specific order of limits (taking $\omega \rightarrow 0$ before $\mathbf{q} \rightarrow 0$ ) is crucial, since the more standard order $\lim _{\omega \rightarrow 0} \lim _{\mathbf{q} \rightarrow 0} \frac{1}{i \omega} \chi_{j_{x}, j_{y}}(\mathbf{q}, \omega)$ yields zero for a translationally invariant system [24]; this subtlety also arises when analyzing edge currents [25-28].

In addition, Eq. (12) was obtained by taking the finite nature of realistic systems into account. In particular, one would obtain an additional factor of $1 / 2$ for a strictly translationally invariant system. Indeed, when deriving Eq. (8), we use that $\left\langle A(\mathbf{q}), B\left(\mathbf{q}^{\prime}\right)\right\rangle \propto \delta_{\mathbf{q},-\mathbf{q}^{\prime}}$ for a strictly infinite translationally invariant system. From this, it follows that $\lim _{\mathbf{q} \rightarrow 0}\langle n(\mathbf{q}) n(\mathbf{q})\rangle=$ 0 and such terms can be discarded. However, for a finite physical system of size $L$, momentum is only defined with a 
resolution $\sim 1 / L$. This means that $\langle n(\mathbf{q}) n(\mathbf{q})\rangle$ starts to become nonzero for $q \lesssim 1 / L$ and in particular $\lim _{\mathbf{q} \rightarrow 0}\langle n(\mathbf{q}) n(\mathbf{q})\rangle=$ $\langle n(\mathbf{0}) n(\mathbf{0})\rangle$ for a finite system, which leads to the extra factor of 2 on the right-hand side of Eq. (12). Physically, it means that a finite system cannot distinguish between a force with a wavelength much greater than the system size from a uniform force. We note that uniform circular shaking $(\mathbf{q}=\mathbf{0})$ can be realized in ultracold-atom experiments. We also point out that Eq. (12) is universal: It can also be derived from KramersKronig relations $[15,19,29,30]$, noting that the excitation rates $\Gamma_{ \pm}(\Omega)$ are related to the power absorbed upon the circular drive $P_{ \pm}(\Omega)=\Omega \Gamma_{ \pm}(\Omega)$.

The Hall conductivity of a superfluid was previously shown to be related to a Chern number; see Refs. [25,26] in the context of chiral ${ }^{3} \mathrm{He}$ superfluids. Using BCS theory, the current-density correlation function can be written as [31]

$$
\chi_{j_{x}, n}(q)=-\sum_{k} \frac{k_{x}}{2 m} \operatorname{tr}\left[\mathcal{G}_{0}(k-q / 2) \mathcal{G}_{0}(k+q / 2) \tau_{3}\right],
$$

where in shorthand notation $k=\left(\mathbf{k}, \omega_{n}\right)$ with $\omega_{n}$ a fermionic Matsubara frequency, and $\mathcal{G}_{0}$ is the BCS Green's function. We have

$$
\mathcal{G}_{0}(k)=\int_{-\infty}^{\infty} \frac{d \omega}{(-\pi)} \frac{\operatorname{Im} \mathcal{G}_{0}(\mathbf{k}, \omega)}{i \omega_{n}-\omega},
$$

where

$$
\begin{aligned}
\operatorname{Im} \mathcal{G}_{0}(\mathbf{k}, \omega)= & -\frac{\pi}{2 E_{\mathbf{k}}}\left(\begin{array}{cc}
\omega+\xi_{\mathbf{k}} & -\Delta_{\mathbf{k}} \\
-\Delta_{\mathbf{k}}^{*} & \omega-\xi_{\mathbf{k}}
\end{array}\right) \\
& \times\left[\delta\left(\omega-E_{\mathbf{k}}\right)-\delta\left(\omega+E_{\mathbf{k}}\right)\right] .
\end{aligned}
$$

Inserting this in Eq. (15) and performing the Matsubara sum yields to first order in $\mathbf{q}$,

$$
\begin{aligned}
\lim _{\omega \rightarrow 0} \chi_{j_{x}, n}(\mathbf{q}, \omega)= & -\frac{i q_{x}}{\mathcal{V}} \sum_{\mathbf{k}} \frac{k_{x}}{2 m} \frac{\operatorname{Im} \Delta_{\mathbf{k}} \partial_{k_{x}} \Delta_{\mathbf{k}}^{*}}{E_{\mathbf{k}}^{3}} \\
& -\frac{i q_{y}}{2 \mathcal{V}} \sum_{\mathbf{k}} \frac{k_{x}}{2 m} \frac{\operatorname{Im} \Delta_{\mathbf{k}} \partial_{k_{y}} \Delta_{\mathbf{k}}^{*}}{E_{\mathbf{k}}^{3}} .
\end{aligned}
$$

The first of these terms vanishes, since the summand is odd in $k_{y}$. This can be seen if we fix the phase of the gap function and look at, for instance, the simple example $\Delta_{\mathbf{k}}=k_{x}+i k_{y}$. With this, it is clear that

$$
\begin{aligned}
\lim _{\mathbf{q} \rightarrow 0} \lim _{\omega \rightarrow 0} \frac{\chi_{j_{x}, n}(\mathbf{q}, \omega)}{i q_{y}} & =\int \frac{d^{2} \mathbf{k}}{8 \pi^{2}} \frac{k_{x}}{m} \frac{\operatorname{Im}\left[\Delta_{\mathbf{k}}^{*} \partial_{k_{y}} \Delta_{\mathbf{k}}\right]}{E_{\mathbf{k}}^{3}} \\
& =\frac{C}{4 \pi}+O\left(\Delta^{2} / \mu^{2}\right),
\end{aligned}
$$

where the last equality is obtained by comparing with the Chern number in Eq. (3). In contrast with the case of Chern insulators, where the Hall conductivity is genuinely topological in the thermodynamic limit [32], the Hall response of the superfluid [Eq. (19)] contains a correction scaling as $O\left(\Delta^{2} / \mu^{2}\right)$. This result was previously related to the fact that the edge current of a chiral $p$-wave superconductor is not strictly topological, as opposed to the presence of edge (Majorana) states [33-35].
Finally, combining Eqs. (12) and (19) yields the central result of this work,

$$
\Delta \Gamma / \mathcal{A}=(1 / 2) \mathcal{E}^{2} C+O\left(\Delta^{2} / \mu^{2}\right),
$$

which shows that the DIR related to the dichroic probe is closely related to the Chern number of the superfluid phase: This observable exhibits a jump proportional to the Chern number to order $O\left(\Delta^{2} / \mu^{2}\right)$ whenever the superfluid enters the topological phase with $C=-1$.

\section{DICHROIC PROBE FOR A TOPOLOGICAL BOSE-FERMI MIXTURE}

We now explore the dichroic probe for a concrete system consisting of a 2D gas of fermionic atoms immersed in a 3D BEC. The fermions interact by exchanging sound modes in the BEC, which leads to an induced attractive interaction and Cooper pairing [7]. Since both the range and strength of the induced interaction can be varied, one can tune the mixture in order to reach a high critical temperature. This makes such a mixture a strong candidate for observing a chiral pairing. Recently, progress towards realizing this goal was reported with the experimental realization of a ${ }^{173} \mathrm{Yb}-{ }^{7} \mathrm{Li}$ mixture [10]. We now analyze how the dichroic probe can be used to detect topological pairing in this specific system.

Due to the finite speed of sound in the BEC, the interaction between the fermions mediated by the bosons is not instantaneous, thus giving rise to retardation effects. The latter are included in the frequency-dependent Eliashberg equations as explained in the Appendix. It has been shown that retardation effects are small when the bosons in the BEC are light compared to the fermions such as for the ${ }^{173} \mathrm{Yb}-{ }^{7} \mathrm{Li}$ mixture [36]. The induced interaction is then close to the static Yukawa form

$$
V(\mathbf{r})=-\frac{a_{\mathrm{eff}}^{2} n_{B} m_{B}}{\pi} \frac{\exp \left(-\sqrt{2} r / \xi_{B}\right)}{r} .
$$

Here, $n_{B}$ and $m_{B}$ is the density and mass of the bosons, $\xi_{B}=$ $1 / \sqrt{8 \pi n_{B} a_{B}}$ is the BEC healing length with $a_{B}$ the bosonboson scattering length, and $a_{\text {eff }}$ is the mixed dimensional Bose-Fermi scattering length.

According to Eq. (7), one should measure the differential rate $\Gamma_{+}-\Gamma_{-}$integrated over all frequencies. However, any real measurement necessarily introduces an upper cutoff frequency $\Omega_{c}$ above which there is no signal [17]. Using Eqs. (12) and (19), the resulting signal reads

$$
\frac{\Delta \Gamma_{\text {trunc }}\left(\Omega_{c}\right)}{\mathcal{A \mathcal { E } ^ { 2 }}} \equiv \int \frac{d^{2} k}{4 \pi} \frac{k_{x}}{m} \frac{\operatorname{Im}\left[\Delta_{\mathbf{k}}^{*} \partial_{k_{y}} \Delta_{\mathbf{k}}\right]}{E_{\mathbf{k}}^{3}} \theta\left(\Omega_{c}-2 E_{\mathbf{k}}\right) \text {. }
$$

The cutoff $\theta\left(\Omega_{c}-2 E_{\mathbf{k}}\right)$ reflects that the probe breaks pairs with energy $2 E_{\mathbf{k}}$ in the long-wavelength limit. We note that $\Delta \Gamma=\lim _{\Omega_{c} \rightarrow \infty} \Delta \Gamma_{\text {trunc }}\left(\Omega_{c}\right)$.

In Fig. 1 , we plot $\Delta \Gamma_{\text {trunc }}\left(\Omega_{c}\right)$ for a ${ }^{7} \mathrm{Li}^{173} \mathrm{Yb}$ mixture with a Bose-Fermi coupling $n_{B}^{1 / 3} a_{\text {eff }}=0.12$, BEC gas parameter $n_{B}^{1 / 3} a_{B}=0.1$, and density ratio $n_{F}^{1 / 2} / n_{B}^{1 / 3}=0.5$, where $n_{F}$ is the 2D Fermi density. These results are obtained by first solving the BCS equations self-consistently at zero temperature and then evaluating the DIR from Eq. (22). The numerical solution indeed confirms the $p$-wave form of pairing $\Delta_{\mathbf{k}}=$ $\Delta_{k} e^{i \phi}$ where $\Delta_{k} \propto k$ for small momenta. 


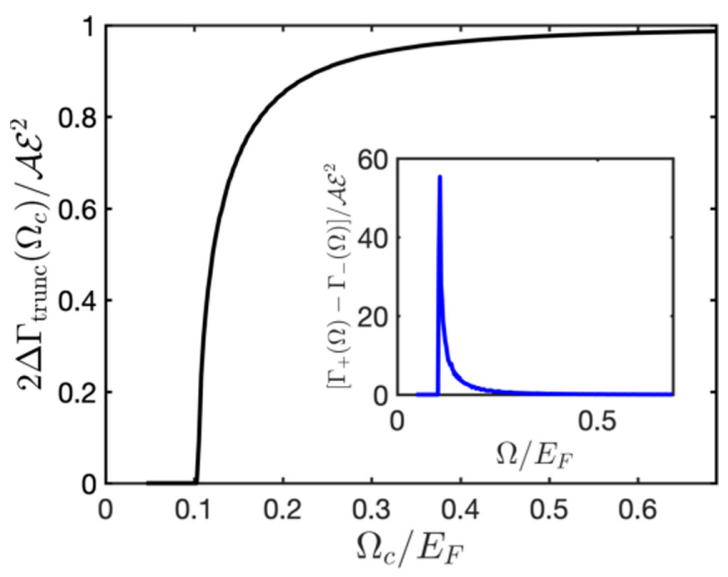

FIG. 1. The differential integrated rate $\Delta \Gamma_{\text {trunc }}(\Omega)$ between a clockwise and counterclockwise perturbation as a function of the cutoff frequency. The inset shows the difference in the heating rates between a clockwise and counterclockwise perturbation as a function of frequency.

The DIR is zero for cutoff frequencies below twice the gap, i.e., for $\Omega_{c} \lesssim 0.1 E_{F}$, where $E_{F}$ is the $2 \mathrm{D}$ Fermi energy, reflecting that there is not enough energy in the probe to break pairs. Above this threshold, the DIR quickly converges towards to the Chern number for $\Omega_{c} \gtrsim E_{F}$. Since $\Delta \simeq 0.05 E_{F} \ll E_{F}$ for this set of parameters, the deviation of $2 \Delta \Gamma / \mathcal{A} \mathcal{E}^{2}$ away from the Chern number is small. We also plot in Fig. 1 the differential rate at a given frequency $\Omega$,

$$
\frac{1}{2}\left[\Gamma_{+}(\Omega)-\Gamma_{-}(\Omega)\right]=\frac{\partial}{\partial \Omega} \Delta \Gamma_{\text {trunc }}(\Omega) .
$$

This difference is large for frequencies just above the threshold given by twice the gap, where the density of states of the superfluid is highest, and Fig. 1 shows that one only needs to measure the difference up to a few times the pairing gap to resolve the Chern number.

One of the appealing features of the Bose-Fermi mixture is that the critical temperature for the 2D superfluid can be tuned to be close to the maximum value $T_{c} / T_{F}=1 / 16$ allowed by BKT theory. Maximizing $T_{c}$ will however also increase the gap and thereby increase corrections to the DIR away from the Chern number as seen from Eq. (20). To analyze this tension, we plot in Fig. 2 the DIR $\Delta \Gamma$ at zero temperature and the critical temperature $T_{c}$ as a function of the gas parameter $n_{B}^{1 / 3} a_{B}$ for $n_{F}^{1 / 2} / n_{B}^{1 / 3}=0.5$ and two different Bose-Fermi interaction strengths. The critical temperature is calculated by combining strong-coupling Eliashberg and BKT theory, which includes the frequency dependence of the gap; see Ref. [7] and the Appendix for details. We see that the critical temperature increases with decreasing gas parameter, reflecting that the range of interaction in Eq. (21), given by the BEC coherence length, increases. The gap consequently also increases, leading to a larger correction term for the DIR away from $\Delta \Gamma=\mathcal{A} \mathcal{E}^{2} \mathcal{C} / 2$. Nevertheless, Fig. 2 shows that there is a significant region where both the DIR is close to the topological value and the critical temperature is close to its maximum value $T_{c} / T_{F}=1 / 16$. Note that we expect our calculation to give a lower bound on the DIR, since BCS theory likely overestimates the gap.

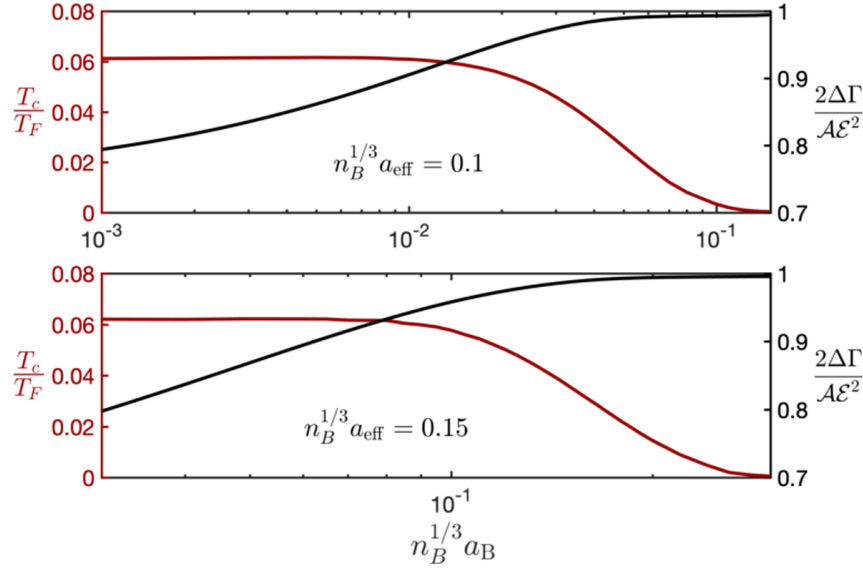

FIG. 2. The critical temperature (red) and the differential integrated rate $\Delta \Gamma$ (black) as a function of the BEC gas parameter $n_{B}^{1 / 3} a_{B}$ for two different Bose-Fermi interaction strengths.

To further illustrate the competition between maximizing the critical temperature and measuring a value of $\Delta \Gamma$ determined by the underlying topology, we plot $\Delta \Gamma$ at zero temperature as a function of $T_{c}$ in Fig. 3 for the same parameters as in Fig. 2. This demonstrates that in order for the dichroic probe to yield a value close to that given by the Chern number, one should cool to around $T \sim 0.06 E_{F}$. Since temperatures down to $T \simeq 0.03 E_{F}$ have been obtained for $2 \mathrm{D}$ Fermi gases [37-39], this is within present day technology making our scheme promising for detecting topological superfluidity. It also shows that a stronger Bose-Fermi interaction strength is slightly more favorable, although the difference between the two interaction strengths is small.

For $T=0$, BCS theory has been shown to be surprisingly accurate even for strong coupling where the Cooper pairs are tightly bound and the system is in the so-called BEC regime [40]. It follows that our calculation of the DIR is reliable even in this regime, where the correction term $O\left(\Delta^{2} / \mu^{2}\right)$ away from the quantized value is large. Any nonzero value however indicates chiral pairing, since the DIR is zero in a phase with time-reversal symmetry. Our scheme thus provides a way to observe the topological phase transition to a trivial phase when $\mu$ becomes negative deep in the BEC regime.

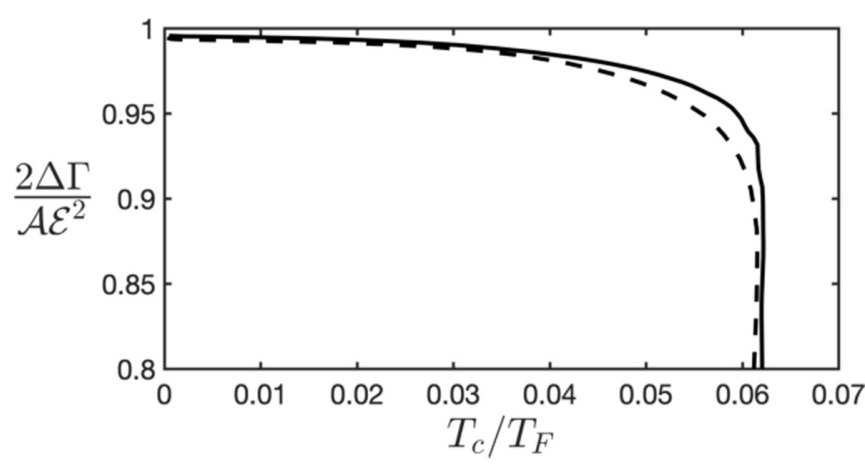

FIG. 3. The DIR as a function of the critical temperature for the same parameters as in Fig. 2. The dashed line corresponds to $n_{B}^{1 / 3} a_{\text {eff }}=0.1$ and the solid line to $n_{B}^{1 / 3} a_{\text {eff }}=0.15$. 


\section{CONCLUSION}

We showed that chiral $p_{x}+i p_{y}$ pairing in a $2 \mathrm{D}$ superfluid can be detected through circular dichroism. Contrary to the case of topological insulators [15], the DIR is not purely dictated by the Chern number due to a correction term scaling as $\Delta^{2} / E_{F}^{2}$, giving rise to a competition between maximizing the critical temperature of the superfluid and observing the Chern number from such a dichroic probe. As a concrete example, we considered an atomic Bose-Fermi mixture. Using a combination of Eliashberg and BKT theory, it was demonstrated that there is in fact a wide range of values for the system parameters where both the critical temperature is high and the dichroic signal is close to the value given by the Chern number. This, combined with the fact that a similar scheme was recently successfully applied to detect topological order in a Chern Bloch band [17], leads to the conclusion that the dichroic probe is a strong candidate for detecting topological $p_{x}+i p_{y}$ pairing in an atomic system.

\section{ACKNOWLEDGMENTS}

J.M.M. and G.M.B. wish to acknowledge the support of the Danish Council of Independent Research-Natural Sciences via Grant No. DFF-4002-00336. Z.W. acknowledges the support by the National Science Foundation of China (Grant No. 11904417) and the Key-Area Research and Development Program of GuangDong Province (Grant No. 2019B030330001). N.G. is supported by the ERC Starting Grant TopoCold, and the Fonds De La Recherche Scientifique (FRS-FNRS, Belgium).

\section{APPENDIX: CALCULATION OF THE SUPERFLUID TRANSITION TEMPERATURE}

Here, we outline the calculation of the superfluid transition temperature for the $2 \mathrm{D}{ }^{173} \mathrm{Yb}$ gas immersed in a $3 \mathrm{D}{ }^{7} \mathrm{Li}$ BEC. First, we solve the following frequency-dependent gap equation at a finite temperature $T$ [36],

$$
\begin{aligned}
\Delta\left(\mathbf{p}, i \omega_{n}\right)= & -T \sum_{m} \int \frac{d \mathbf{q}}{(2 \pi)^{2}} V_{\text {ind }}\left(\mathbf{p}-\mathbf{q}, i \omega_{n}-i \omega_{m}\right) \\
& \times \frac{\Delta\left(\mathbf{q}, i \omega_{m}\right)}{\omega_{m}^{2}+\mathcal{E}^{2}\left(\mathbf{q}, i \omega_{m}\right)},
\end{aligned}
$$

where $\mathcal{E}\left(\mathbf{q}, i \omega_{m}\right)=\sqrt{\xi_{\mathbf{q}}^{2}+\left|\Delta\left(\mathbf{q}, i \omega_{m}\right)\right|^{2}}$. Here, the frequencydependent induced interaction $V_{\text {ind }}\left(\mathbf{q}, i \omega_{v}\right)$ is given by

$$
\begin{aligned}
V_{\text {ind }}\left(\mathbf{q}, i \omega_{\nu}\right)= & -n_{B} m_{B} g^{2}\left[\left(\frac{1}{\kappa_{+}}+\frac{1}{\kappa_{-}}\right)\right. \\
& \left.+\frac{1}{\sqrt{1-\left(\omega_{\nu} / g_{B} n_{B}\right)^{2}}}\left(\frac{1}{\kappa_{+}}-\frac{1}{\kappa_{-}}\right)\right],
\end{aligned}
$$

where $\kappa_{ \pm}=\sqrt{2 m_{B} g_{B} n_{B}\left[1 \pm \sqrt{1-\left(\omega_{\nu} / g_{B} n_{B}\right)^{2}}\right]+\mathbf{q}^{2}}$. Along with a number equation, this constitutes the Eliashberg equations of the superfluid [31].

Since the Fermi system is $2 \mathrm{D}$, the superfluid transition is driven by vortex-antivortex proliferation and the critical temperature $T_{\mathrm{BKT}}$ is determined by the Kosterlitz-Thouless condition [7]

$$
T_{\mathrm{BKT}}=\frac{\pi}{8 m_{F}^{2}} \rho_{s}\left(\left\{\Delta\left(i \omega_{n}\right)\right\}, T_{\mathrm{BKT}}\right) .
$$

Here, $\rho_{s}$ is the superfluid mass density and is a function of the gap parameters and the temperature. Neglecting the renormalization of the interaction between vortex pairs, $\rho_{s}$ can be estimated as

$$
\rho_{s}=\rho_{0}+\frac{T}{2} \sum_{n} \int \frac{d \mathbf{p}}{(2 \pi)^{2}} p^{2} \frac{\mathcal{E}^{2}\left(\mathbf{p}, i \omega_{n}\right)-\omega_{n}^{2}}{\left[\omega_{n}^{2}+\mathcal{E}^{2}\left(\mathbf{p}, i \omega_{n}\right)\right]^{2}},
$$

where $\rho_{0}=m_{F} n_{F}$. Solving Eq. (A3) self-consistently using Eq. (A4) and the frequency-dependent gap parameters obtained from Eq. (A1), we obtain the superfluid transition temperatures shown in the main text.
[1] C. Nayak, S. H. Simon, A. Stern, M. Freedman, and S. Das Sarma, Rev. Mod. Phys. 80, 1083 (2008).

[2] S. Nadj-Perge, I. K. Drozdov, J. Li, H. Chen, S. Jeon, J. Seo, A. H. MacDonald, B. A. Bernevig, and A. Yazdani, Science 346, 602 (2014).

[3] R. M. Lutchyn, E. P. A. M. Bakkers, L. P. Kouwenhoven, P. Krogstrup, C. M. Marcus, and Y. Oreg, Nat. Rev. Mater. 3, 52 (2018).

[4] C. W. Hicks, J. R. Kirtley, T. M. Lippman, N. C. Koshnick, M. E. Huber, Y. Maeno, W. M. Yuhasz, M. B. Maple, and K. A. Moler, Phys. Rev. B 81, 214501 (2010).

[5] A. P. Mackenzie, T. Scaffidi, C. W. Hicks, and Y. Maeno, npj Quantum Mater. 2, 40 (2017).

[6] S. A. Kivelson, A. C. Yuan, B. Ramshaw, and R. Thomale, npj Quantum Mater. 5, 43 (2020).

[7] Z. Wu and G. M. Bruun, Phys. Rev. Lett. 117, 245302 (2016).

[8] J. M. Midtgaard, Z. Wu, and G. M. Bruun, Phys. Rev. A 94, 063631 (2016).
[9] J. M. Midtgaard, Z. Wu, and G. M. Bruun, Phys. Rev. A 96, 033605 (2017).

[10] F. Schäfer, N. Mizukami, P. Yu, S. Koibuchi, A. Bouscal, and Y. Takahashi, Phys. Rev. A 98, 051602(R) (2018).

[11] E. Grosfeld, N. R. Cooper, A. Stern, and R. Ilan, Phys. Rev. B 76, 104516 (2007).

[12] N. R. Cooper, J. Dalibard, and I. B. Spielman, Rev. Mod. Phys. 91, 015005 (2019).

[13] G. Möller, N. R. Cooper, and V. Gurarie, Phys. Rev. B 83, 014513 (2011).

[14] M. Gong, G. Chen, S. Jia, and C. Zhang, Phys. Rev. Lett. 109, 105302 (2012).

[15] D. T. Tran, A. Dauphin, A. G. Grushin, P. Zoller, and N. Goldman, Sci. Adv. 3, e1701207 (2017).

[16] D.-T. Tran, N. R. Cooper, and N. Goldman, Phys. Rev. A 97, 061602(R) (2018).

[17] L. Asteria, D. T. Tran, T. Ozawa, M. Tarnowski, B. S. Rem, N. Fläschner, K. Sengstock, N. Goldman, and C. Weitenberg, Nat. Phys. 15, 449 (2019). 
[18] M. Schüler and P. Werner, Phys. Rev. B 96, 155122 (2017).

[19] C. Repellin and N. Goldman, Phys. Rev. Lett. 122, 166801 (2019).

[20] P. W. Klein, A. G. Grushin, and K. L. Hur, arXiv:2002.01742.

[21] P. W. Anderson and P. Morel, Phys. Rev. 123, 1911 (1961).

[22] J. P. Lu and W. Barford, Phys. Rev. B 44, 5263 (1991).

[23] N. Read and D. Green, Phys. Rev. B 61, 10267 (2000).

[24] G. Giuliani and G. Vignale, Quantum Theory of the Electron Liquid (Cambridge University Press, Cambridge, U.K., 2005).

[25] G. E. Volovik, JETP Lett. 55, 368 (1992).

[26] G. E. Volovik, Sov. Phys. - JETP 67, 1804 (1988).

[27] J. Goryo and K. Ishikawa, Phys. Lett. A 246, 549 (1998).

[28] M. Stone and R. Roy, Phys. Rev. B 69, 184511 (2004).

[29] H. S. Bennett and E. A. Stern, Phys. Rev. 137, A448 (1965).

[30] I. Souza and D. Vanderbilt, Phys. Rev. B 77, 054438 (2008).

[31] G. Mahan, Many-Particle Physics (Kluwer Academic/Plenum Publishers, New York, 2000).
[32] D. J. Thouless, M. Kohmoto, M. P. Nightingale, and M. den Nijs, Phys. Rev. Lett. 49, 405 (1982).

[33] E. Taylor and C. Kallin, Phys. Rev. Lett. 108, 157001 (2012).

[34] W. Huang, E. Taylor, and C. Kallin, Phys. Rev. B 90, 224519 (2014).

[35] W. Huang, S. Lederer, E. Taylor, and C. Kallin, Phys. Rev. B 91, 094507 (2015).

[36] J. J. Kinnunen, Z. Wu, and G. M. Bruun, Phys. Rev. Lett. 121, 253402 (2018).

[37] N. Luick, L. Sobirey, M. Bohlen, V. P. Singh, L. Mathey, T. Lompe, and H. Moritz, Science 369, 89 (2020).

[38] L. Sobirey, N. Luick, M. Bohlen, H. Biss, H. Moritz, and T. Lompe, arXiv:2005.07607.

[39] M. G. Ries, A. N. Wenz, G. Zürn, L. Bayha, I. Boettcher, D. Kedar, P. A. Murthy, M. Neidig, T. Lompe, and S. Jochim, Phys. Rev. Lett. 114, 230401 (2015).

[40] P. Pieri, L. Pisani, and G. C. Strinati, Phys. Rev. B 72, 012506 (2005). 